\title{
POST-ARC CHARACTERISTICS OF HIGH-CURRENT ARCS IN VACUUM CIRCUIT-BREAKERS
}

\author{
N. WenZEL ${ }^{a, *}$, W. HAAS ${ }^{b}$ \\ a Siemens AG, Corporate Technology, Research in Energy and Electronics, 91050 Erlangen, Germany \\ ${ }^{b}$ Retired employee of Siemens AG, Zentralabteilung Forschung und Entwicklung, 91050 Erlangen, Germany \\ * norbert.wenzel@siemens.com
}

\begin{abstract}
The post-arc (PA) characteristics of vacuum arcs in transverse magnetic field contacts are studied for short-circuit currents of up to $123 \mathrm{kA}$ peak and transient recovery voltages below $875 \mathrm{~V}$. The measured PA currents are interpreted in terms of an Electric Resistance Model and the models of Andrews-Varey, Langmuir-Child, and Slepian-Schmelzle. Whereas in the late PA period, the calculations do not agree well with the measurements, the PA behavior is well described in the early period after current-zero. It is concluded that the PA discharge is amplified by ionization of metal vapor particles in the boundary sheath due to electron impact.
\end{abstract}

Keywords: vacuum arc, vacuum circuit-breaker, transient recovery voltage, post-arc current, boundary sheath, transverse magnetic field contact.

\section{Introduction}

Vacuum arcs are successfully applied in switching technology to interrupt high short-circuit (SC) currents. The transient recovery voltage (TRV) of the electrical grid after current-zero removes the residual electric charge carriers of the switching arc plasma from the contact gap region. This results in a "post-arc current" (PAC) that is still flowing after vacuum arc extinction (e.g. early papers $[1,2]$ or recent papers $[3-5]$ ). The PAC behavior of the contact gap is of central importance for the breaking performance of a vacuum interrupter.

Due to the fast removal of the electrons within microseconds after current-zero, the (heavy) metal ions (copper and chromium) establish a positive space charge sheath near the post-arc (PA) cathode, the "post-arc boundary sheath", which is expanding towards the PA anode. The movement of the ions and the ionization of neutral metal vapor particles within the sheath sensitively influence the PAC characteristics.

Former published studies of the PAC behavior were restricted to medium $\mathrm{SC}$ currents ranging from $36 \mathrm{kA}$ rms to $60 \mathrm{kA}$ rms $[1,2,6-8]$. They were mostly concerned with high TRV's up to $80 \mathrm{kV}[6,8]$ and $126 \mathrm{kV}$ [9]. Studies concerned with low TRV's $(\leq 960 \mathrm{~V})$ reported in [10] were restricted to medium SC currents ( $\leq 50 \mathrm{kA} \mathrm{rms}$ ) and focused to axial magnetic field contacts.

In this contribution, the PAC behavior of a transverse magnetic field (TMF) contact in a high-current low-voltage vacuum interrupter is investigated. Experiments in a synthetic test circuit and physical models of the PA boundary sheath are described, and analytical computations are used to interpret the measurements. The studies resume and critically revalidate

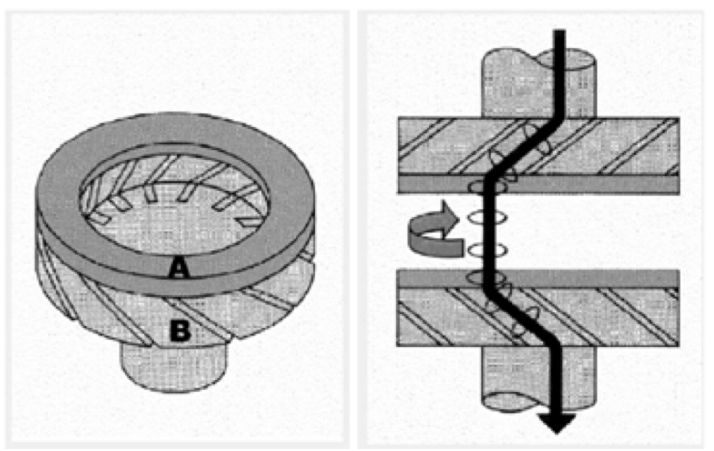

Figure 1. TMF contact [18]. Left: Cu contact piece (B) with $\mathrm{CuCr}$ contact ring $(A)$; contact diameter $=$ $90 \mathrm{~mm}$; width of contact ring $=18 \mathrm{~mm}$. Right: Current path and moving constricted vacuum arc.

investigations, that had been reported in [11], but not yet published in written form.

\section{Experiments}

The experiments are performed with a conventional high-current low-voltage vacuum interrupter. A moving constricted metal vapor arc is drawn by separating the electrodes of a cup-shaped (contrate) TMF contact to a gap of $3 \mathrm{~mm}$ at a velocity of typically $1 \mathrm{~m} / \mathrm{s}$. The contact system is sketched in Figure 1.

Single sinusoidal SC currents (AC half period $12 \mathrm{~ms}$ ) with amplitudes of up to $123 \mathrm{kA}$ peak corresponding to $87 \mathrm{kA}$ rms are produced by a capacitor bank, supplemented by a DC pre-current of $100 \mathrm{~A}$. Recovery voltages with the same relative step response waveform and varying steady-state values ranging from $U_{\mathrm{W}}=385 \mathrm{~V}$ to $875 \mathrm{~V}$ (absolute values) are generated in a synthetic test circuit. Wide-band transformers (Pearson Electronics) are used to monitor the tremendously differing current amplitudes in the SC path 


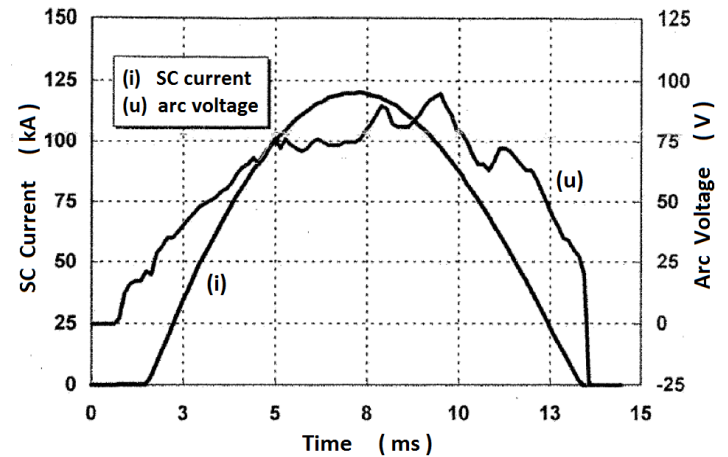

Figure 2. Measured time evolutions of $S C$ current and vacuum arc voltage. Time $t=0$ : start of DC precurrent; $t=1 \mathrm{~ms}$ : contact separation; $t=2 \mathrm{~ms}$ : start of current half-period.

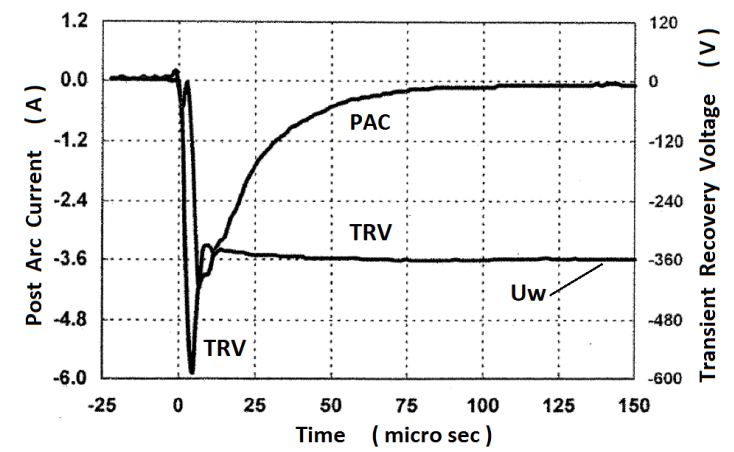

Figure 3. Measured time evolutions of applied TRV $\left(U_{\mathrm{S}}\right)$ and resulting PAC current-zero. After the initial voltage peak $(-595 \mathrm{~V})$, the TRV converges to the steadystate value $(-360 \mathrm{~V})$ denoted as $U_{\mathrm{W}}$.

and the PAC path of the synthetic circuit. Figure 2 displays a typical example of the measured time evolutions of SC current and arc voltage. Figure 3 shows a typical example of the measured time evolutions of the TRV (denoted as $U_{\mathrm{S}}$ in the next chapters) and the resulting PAC. The relative TRV waveform remains the same, when the steady-state voltage $U_{\mathrm{W}}$ changes.

\section{Modeling}

Different one-dimensional analytical models are used to describe the expansion of the PA boundary sheath within the contact gap towards the (new) PA anode under the impact of the TRV. The sheath expansion is sketched in Figure 4. Though primarly appropriate for diffuse vacuum arcs in axial magnetic field contacts, one-dimensional computation is also applicable to TMF contacts, since constricted vacuum arcs become diffuse near current-zero (below $15 \mathrm{kA}$ ).

The models formulate relations between the instantaneous values of the PAC density, $j$, the expansion velocity of the sheath boundary, $\partial s / \partial t$, and the TRV, $U_{\mathrm{S}}$. The expansion velocity is linked to the PAC density via Equation (1). The quantities $q$ and $n_{0}$, respectively, denote the electric charge and the density of the ions in the transition zone between boundary

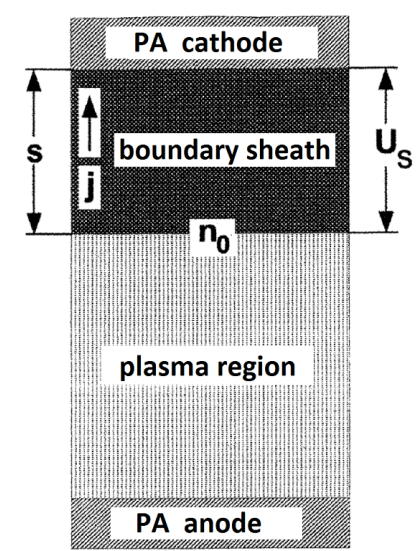

Figure 4. Sketch of PA boundary sheath (width s) expanding from $P A$ cathode towards $P A$ anode under the impact of the TRV $\left(U_{S}\right) . j=P A C$ density; $n_{0}=$ ion density in transition zone between boundary sheath and PA plasma region. Details see text.

sheath and PA plasma region. The function $g\left(U_{\mathrm{S}}\right)$ reflects the influence of $U_{\mathrm{S}}$ and is specified by the respective model of the boundary sheath.

$$
\frac{\partial s}{\partial t}=\frac{j}{q n_{0}} g\left(U_{\mathrm{S}}\right)
$$

The measured PAC evolutions are interpreted in terms of an "Electric Resistance Model" of the sheath developed in [11]. And they are compared with simulations based on the sheath models of Andrews-Varey, Langmuir-Child, Slepian, and Schmelzle.

The Transition Model of Andrews-Varey [12, 13], and the model according to the Langmuir-Child Law [14] describe the "free" flight of the ions in the boundary sheath. The model of Andrews-Varey takes the displacement and the conduction current into account, and it assumes only small changes of the ion parameters over a transition of the boundary sheath. The model yields Equation (2), an implicit relation between the TRV, $U_{\mathrm{S}}$, and the PAC density, $j$, both linked by the width $s$ of the boundary sheath ( $M$ : ion mass, $\varepsilon_{0}$ : vacuum permittivity constant).

$$
\begin{aligned}
s^{2} & =\frac{4}{9}\left(\frac{\varepsilon_{0} M}{2 q^{4}} \frac{j^{2}}{n_{0}^{3}}\right)\left[\left(1+\frac{2 q^{3}}{M} U_{\mathrm{S}} \frac{n_{0}^{2}}{j^{2}}\right)^{\frac{2}{3}}\right. \\
& \left.+3 \frac{2 q^{3}}{M} U_{\mathrm{S}} \frac{n_{0}^{2}}{j^{2}}-1\right]
\end{aligned}
$$

The model of Langmuir-Child results in Equation (3), an explicit relation between $U_{\mathrm{S}}$ and $j$. It is derived from Equation (2) by neglecting the kinetic energy of the ions, $0.5 M(\partial s / \partial t)^{2}$, against the energy they gain due to the TRV, $q U_{\mathrm{S}}$.

$$
U_{\mathrm{S}}=\left(\frac{9}{4 \varepsilon_{0}}\right)^{\frac{2}{3}}\left(\frac{M}{2 q}\right) s^{\frac{4}{3}} j^{\frac{2}{3}}
$$


The models of Slepian and Schmelzle [15, 16], and the "Electric Resistance Model" [11] describe the motion of the ions under the assumption that the motion is dominated by collisions in the boundary sheath. The models neglect the displacement current and yield explicit relations between $U_{\mathrm{S}}$ and $j$.

The models of Slepian and Schmelzle, respectively, result in the (precise) first and the (approximate) second term of Equation (4) in terms of the mobility of the ions, $\mu$, whereupon the (second) term according to Slepian neglects the summands proportional to $j /\left(q n_{0}\right)^{2}$ by assuming high ion densities $n_{0}$ in the transition zone.

$$
\begin{aligned}
U_{\mathrm{S}} & =\frac{2}{3}\left(\frac{2 j}{\varepsilon_{0} \mu}\left[\left(\frac{j \varepsilon_{0}}{2 q^{2} n_{0}^{2}}+s\right)^{\frac{3}{2}}-\left(\frac{j \varepsilon_{0}}{2 q^{2} n_{0}^{2}}\right)^{\frac{3}{2}}\right]\right)^{\frac{1}{2}} \\
& \approx \frac{2}{3}\left(\frac{2}{\varepsilon_{0}} \frac{j}{\mu} s^{\frac{3}{2}}\right)^{\frac{1}{2}}
\end{aligned}
$$

The "Electric Resistance Model" reflects the local Ohm Law. It connects the PAC density, $j$, with the average electric field, $U_{\mathrm{S}} / s$, in the boundary sheath via Equation (5) in terms of the ion mobility, $\mu=$ $\sigma /(q\langle n\rangle)$, where $\langle n\rangle$ denotes the average ion density in the boundary sheath.

$$
U_{\mathrm{S}}=\frac{j s}{q\langle n\rangle \mu}
$$

The described models are applicable provided that evaporation on the PA cathode is negligible. In the case of TMF arcs, particularly at higher currents, the surface of the (old) anode during SC current flow, i.e. the (new) PA cathode, could be largely molten supplying ions even after SC current-zero into the switching gap. The model descriptions are thus justified for switching operations well below the SC breaking limit of a TMF contact system.

\section{Results and discussion}

The experiments document that high SC currents, large SC arcing times, and high TRV's cause high values of PAC amplitude and residual electric PA charge, the time integral of the PAC. Figure (5) shows the peak PAC amplitude and the residual PA charge as function of the steady-state value of the TRV waveform, $U_{\mathrm{W}}$, for a current of $87 \mathrm{kA} \mathrm{rms}$ and an arcing time of $10 \mathrm{~ms}$. The PA quantities measured for successful interruptions can be well fitted to an exponential function. The PA quantities referring to SC failures exhibit significantly higher amplitudes than those for successful switching operations.

Figure (6) shows a comparison of the measured time evolutions of TRV (points) and PAC (points) with simulations (lines) based on the "Electric Resistance Model" and the models of Langmuir-Child and
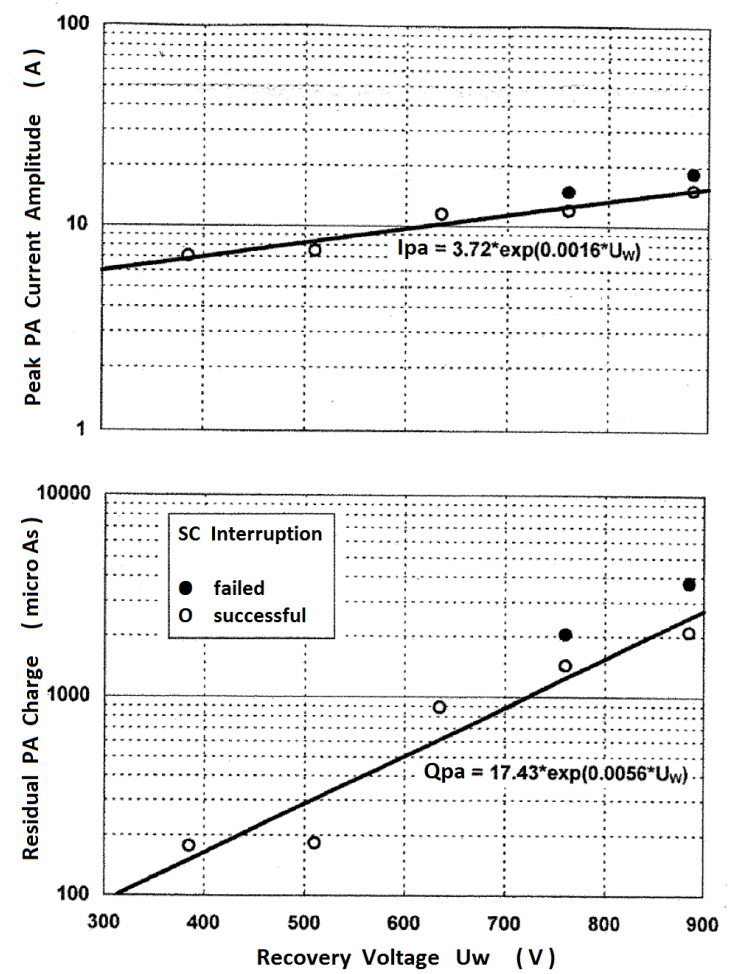

Figure 5. Measured average values of peak PAC amplitude $I_{\mathrm{PA}}$ (top) and residual PA charge $Q_{\mathrm{PA}}$ (bottom) as function of TRV $U_{\mathrm{W}}$. Open circles: successful interruptions, full circles: failed interruptions, solid line: fit to successful interruptions. SC current $=87 k A_{\mathrm{rms}}$, arcing time $=10 \mathrm{~ms}$.

Slepian-Schmelzle. The model parameter is the average plasma density, $n$, in the contact gap after SC arc extinction. The simulations according to AndrewsVarey (not shown here) and Langmuir-Child yield nearly identical results.

In the early PA period (between $1 \mu \mathrm{s}$ and $4 \mu \mathrm{s}$ after current-zero), the simulations display a good description of the measured evolution of the PAC, if an average plasma density of several $10^{19} \mathrm{~m}^{-3}$ is assumed after the extinction of the SC arc. In the late PA period ( $>5 \mu \mathrm{s}$ ) however, the calculations do not agree well with the experiment. This finding indicates that the PA discharge in the contact gap after high-current interruption is amplified by ionization of neutral metal vapor particles in the boundary sheath due to electron impact. The PA amplification starts at sufficiently broad boundary sheath, even under the influence of the applied low recovery voltages $(<1000 \mathrm{~V})$. The ionizing electrons in the boundary sheath can be generated at the PA cathode by thermofield emission ("hot spots") and by secondary emission (ion impact). These physical processes are not described by the used simulation models. In the very early PA period $(<1 \mu \mathrm{s})$, the experimental PAC signals indicate that high-energy particles are penetrating the boundary sheath at the very beginning of sheath development. This phenomenon reported by [17], e.g., is also not described by the used simulation models 

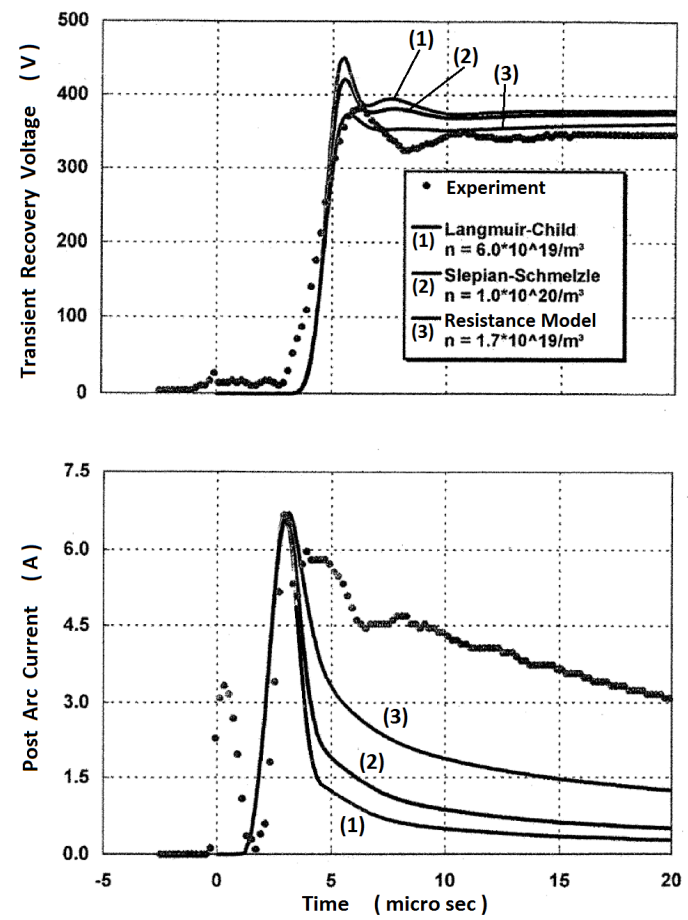

Figure 6. Comparison of measured time evolutions of TRV (points) and PAC (points) with simulations (lines). Boundary sheath models: Langmuir-Child (1), Slepian-Schmelzle (2), and Electric Resistance Model (3). $n=$ plasma density in contact gap after $S C$ arc extinction. Time $t=0: S C$ current-zero. SC current $=87 \mathrm{kA} A_{r m s}$, arcing time $=10 \mathrm{~ms}$.

\section{Conclusion}

In this contribution, the PA characteristics of constricted vacuum arcs moving in a small-gap cupshaped TMF contact system $(\mathrm{CuCr})$ have been investigated for $\mathrm{SC}$ currents $(42 \mathrm{~Hz})$ of up to $123 \mathrm{kA}$ peak and for TRV's below $875 \mathrm{~V}$ (steady-state values). The measured PAC evolutions are strongly related to SC current amplitude, arcing time, and applied TRV. The experiments are compared with simulations of the PA boundary sheath based on the models of AndrewsVarey, Langmuir-Child, and Slepian-Schmelzle, and on an "Electric Resistance Model". In the early PA period (between $1 \mu \mathrm{s}$ and $4 \mu \mathrm{s}$ after SC current-zero), the simulations display a good description of the PAC evolution. In the very early (below $1 \mu \mathrm{s}$ ) and in the late PA period (later than $5 \mu$ s after SC current-zero), the calculations do not agree well with the experiment.

The following conclusions can be drawn: After highcurrent interruption, the PA discharge in the contact gap is amplified by ionization of neutral metal vapor particles in the boundary sheath due to electron impact. The ionizing electrons in the boundary sheath are generated at the PA cathode by thermo-field emission and by secondary emission. High-energy particles are penetrating the boundary sheath at the very beginning of sheath development.

Diagnosing and one-dimensional analytical modeling of the PA behavior allow the evaluation of with- stand voltage characteristics and re-ignition behavior of a small-gap TMF vacuum interrupter contact, at least in a crucial time interval after SC interruption. This offers the possibility of a purposeful optimization of high-current vacuum circuit-breakers without applying complex multidimensional numerical simulation methods.

\section{References}

[1] K. Matsumoto. Post arc current after high current interruption in vacuum. In Proc. 11th ISDEIV, Berlin, vol.2, sections 3-6, 1984.

[2] S. Yanabu et al. Post arc current of vacuum interrupters. IEEE Trans. Power Apparatus and Systems, 104(1):166-172, 1985. doi:10.1109/TPAS.1985.318890.

[3] G. Ge, X. Cheng, M. Liao, X. Duan, and J. Zou. Vacuum arcs and postarc characteristic of vacuum interrupters with external AMF at current zero. IEEE Trans. Plasma Sci., 46:1003-1009, 2018. doi:10.1109/TPS. 2018. 2816028.

[4] Y. Mo, Z. Shi, J. Li, S. Jia, and L. Wang. Experimental research of post-arc currents in vacuum circuit breakers. In Proc. 28th ISDEIV, Greifswald, volume 1, pages 193-196, 2018.

[5] A. Shemshadi. Modeling of plasma dispersion process in vacuum interrupters during postarc interval based on FEM. IEEE Trans. Plasma Sci., 47(1):647-653, 2019. doi:10.1109/TPS. 2018.2879519.

[6] K.-D. Weltmann, E. Huber, and K. Froehlich. Arc memory effect on postarc current in vacuum circuit breaker - simulation and experiment. In Proc. 18th ISDEIV, Eindhoven, pages 545-548, 1998. doi:10.1109/DEIV.1998.738710.

[7] S. Hakamata, Y. Kurosawa, K. Natsui, K. Hirasawa, and K. Kurita. Post arc current of vacuum interrupter after large current interruption. IEEE Trans. Power Delivery, 3(4):1692-1697, 1988. doi:10.1109/61.193973.

[8] K. Steinke, M. Lindmayer, and K.-D. Weltmann. Post-arc currents of vacuum interrupters with axial magnetic field contacts under high current and voltage stress. In Proc. 19th ISDEIV, Xi'an, pages 475-480, 2000. doi:10.1109/DEIV.2000.879030.

[9] E. Dullni, E. Schade, and B. Gellert. Dielectric recovery of vacuum arcs after strong anode spot activity. IEEE Trans. Plasma Sci., 15(5):538-544, 1987. doi:10.1109/TPS.1987.4316750.

[10] D. Pavelescu, S. Nitu, G. Dumitrescu, and P. Anghelita. Post-arc current in low-voltage vacuum circuit breaker: Measurements and physical peculiarities. IEEE Trans. Plasma Sci., 31(5):869-876, 2003. doi:10.1109/TPS.2003.818421.

[11] W. Haas and N. Wenzel. Das Nachstromverhalten stromstarker Metalldampf-Lichtboegen in Vakuum (The post-arc current behavior of high-current metal vapor arcs in vacuum). In Fachtagung der Deutschen Gesellschaft für Plasmatechnologie, Bochum, Poster, 1996. 
[12] J. Andrews and R. Varey. Sheath growth in a low pressure plasma. The Physics of Fluids, 14:339-343, 1971. doi:10.1063/1.1693433.

[13] R. Holmes and S. Yanabu. Post-arc current mechanism in vacuum interrupters. J. Phys. D: Appl. Phys., 6(10):1217-1231, 1973. doi:10.1088/0022-3727/6/10/306.

[14] S. Brown. Introduction to Electrical Discharges in Gases. Chapter 12, pp. 200-225. Wiley series in plasma physics. John Wiley and Sons, New York, 1966.

[15] J. Slepian. Extinction of an A-C arc. Transactions on American Institute of Electric Engineering (AIEE), 47(4):1398-1408, 1928.

doi:10.1109/T-AIEE.1928.5055155.
[16] M. Schmelzle. Grenzen der Selbstloeschung kurzer Lichtbogenstrecken bei Wechselstrombelastung (Limits of self-extinction of short arc gaps under AC stress). PhD Thesis, Technical University of Braunschweig, 1968.

[17] X. Li, J. Zou, W. Dong, D. Liang, and T. Qin. Experiment research on post-arc current in DC vacuum circuit breakers. In Proc. 4th Int. Conf. Electric Power Equipment - Switching Technology, Xi'an, pages 375-378, 2017. doi:10.1109/ICEPE-ST.2017.8188867.

[18] D. W. Branston, W. Haas, W. Hartmann, R. Renz, and N. Wenzel. Thermodynamic model of moving vacuum arcs on contrate contacts. In Proc. 22nd ISDEIV, Matsue, volume 1, pages 277-280, 2006. doi:10.1109/DEIV.2006.357285. 